\title{
Time course of cigarette smoke-induced pulmonary inflammation in mice
}

\author{
A.I. D'hulst, K.Y. Vermaelen, G.G. Brusselle, G.F. Joos and R.A. Pauwels
}

\begin{abstract}
Inflammation of the airways and lung parenchyma plays a major role in the pathogenesis of chronic obstructive pulmonary disease. In the present study a murine model of tobacco smoke-induced emphysema was used to investigate the time course of airway and pulmonary inflammatory response, with a special emphasis on pulmonary dendritic cell (DC) populations.
\end{abstract}

Groups of mice were exposed to either cigarette smoke or to control air for up to 24 weeks. In response to cigarette smoke, inflammatory cells (i.e. neutrophils, macrophages and lymphocytes) progressively accumulated both in the airways and lung parenchyma of mice. Furthermore, a clear infiltration of DCs was observed in airways (10-fold increase) and lung parenchyma (1.5-fold increase) of cigarette-exposed mice at 24 weeks.

Flow cytometric analysis of bronchoalveolar lavage (BAL) DCs of smoke-exposed mice showed upregulation of major histocompatability complex II molecules and costimulatory molecules CD40 and CD86, compared with BAL DCs of air-exposed mice. Morphometric analysis of lung histology demonstrated a significant increase in mean linear intercept and alveolar wall destruction after 24 weeks of smoke exposure.

In conclusion, the time course of the changes in inflammatory and dendritic cells in both bronchoalveolar lavage and the pulmonary compartment of cigarette smoke-exposed mice was carefully characterised.

KEYWORDS: Chronic obstructive pulmonary disease, cigarette smoke, dendritic cell, emphysema, macrophage, neutrophil

hronic obstructive pulmonary disease (COPD) is a disease state characterised by the progressive development of airflow limitation that is not fully reversible [1]. Inflammation of the airways and the lungs is thought to play a major role in the pathogenesis of COPD [2-5]. The progression of COPD is strongly associated with infiltration of the small airway walls by inflammatory cells and the accumulation of inflammatory mucous exudates in the lumen [6]. A major risk factor for COPD is cigarette smoking. However, the molecular and cellular basis of the cigarette smoke (CS)-induced inflammation in COPD is still unclear.

The primary host defence mechanisms of the lungs against exposure to toxic gases and particles are the innate and adaptive inflammatory immune responses. The innate defence system of the lung is provided by the epithelial barrier and the acute inflammatory response which follows tissue injury, including the recruitment and activation of neutrophils, eosinophils and macrophages [7]. In contrast, the adaptive immune response is dependent upon B- and
T-lymphocytes (CD4+ and CD8+), and has a longstanding memory for previous damage. Detailed histological analysis of surgically resected lung tissue from patients with various clinical stages of COPD has clearly demonstrated a progressive infiltration of the small airway wall by both innate and adaptive immune cells. Interestingly, as COPD progresses, the percentage of airways containing lymphoid follicles increases, suggesting the involvement of antigenpresenting cells in the organisation of lymphocytes into follicles in patients with severe COPD [6].

Dendritic cells (DCs) are not only specialised antigen-presenting cells, they are also critically located in the airways and the lung parenchyma at the interface between the inhaled air and the lung [8]. The DC is a major sensor of danger and reacts to any disturbance of the mucosal surface by initiating an innate immune response and controlling the development of a specific immune reaction [8-13]. As DCs are able to link innate and adaptive immunity, and since both components of the immune system are augmented in the
AFFILIATIONS

Dept of Respiratory Diseases, Ghent University Hospital, Ghent, Belgium.

CORRESPONDENCE

A.I. D'hulst

Dept of Respiratory Diseases

Ghent University Hospital

De Pintelaan 185

B-9000 Ghent

Belgium

Fax: 3292402341

E-mail: an.dhulst@UGent.be

Received:

August 132004

Accepted after revision:

April 092005

\section{SUPPORT STATEMENT}

This study was supported by the fund for Scientific Research in Flanders Project (grant number G.0011.03) and a Concerted Research Initiative of the Ghent University (project number G0A 1251504).

European Respiratory Journal Print ISSN 0903-1936 Online ISSN 1399-3003 
airways of patients with COPD, the current authors hypothesised that pulmonary DC numbers would increase due to CS exposure and that DCs of smoke-exposed mice would show signs of activation. Therefore, the airway and pulmonary inflammatory response, and, more specifically, the DC response to tobacco-smoke exposure in a mouse model of CS-induced emphysema was studied [14].

\section{METHODS}

\section{Animals}

Male C57Bl/ 6 mice, 6-8-week old were purchased from Harlan (Zeist, The Netherlands). The local Ethics Committee (Ghent, Belgium) approved all in vivo manipulations.

\section{Experimental design}

Mice were exposed to the smoke of five cigarettes (Reference Cigarette 1R3; University of Kentucky, Lexington, KY, USA) q.i.d. with 30 min smoke-free intervals. The animals were exposed 5 days per week for up to 24 weeks. The control group was exposed to air. At days 1, 3, 7 and $14(n=6$; short-term smoke exposure) and at weeks 4, 12 and $24(\mathrm{n}=5$; long-term) mice were sacrificed. These experiments were repeated three times.

\section{Tobacco smoke chamber}

Mice were exposed to CS, using a smoking apparatus (D. Kobayashi, Washington University Medical Center, WA, USA) with the chamber adapted for a group of mice (chamber volume $=7,500 \mathrm{~cm}^{3}$ ). An optimal smoke/air ratio of 1:12 was obtained [15].

\section{Bronchoalveolar lavage}

To perform bronchoalveolar lavage (BAL), animals were sacrificed and a tracheal cannula was inserted. Three times via the trachea, $1 \mathrm{~mL}$ of Hank's balanced salt solution (HBSS; GibcoBRL, Carlsbad, CA, USA), free of ionised calcium and magnesium but supplemented with 0.05 mM EDTA (Sigma, St Louis, MO, USA), was instilled and recovered by gentle manual aspiration. The recovered BAL fluid was centrifuged, the cell pellet washed twice and finally resuspended in $1 \mathrm{~mL}$ of HBSS. The total cell count was performed in a Bürker chamber (Novolab, Geraardsbergen, Belgium) and the differential cell counts were performed on cytocentrifuged preparations (Cytospin 2; Shandon Ltd, Runcorn, UK) after staining with May-Grünwald-Giemsa.

\section{Preparation of lung single cell suspensions}

Following BAL, the pulmonary and systemic circulation was rinsed. One lung was used for histology, the other was used for the preparation of a cell suspension as detailed previously [16]. Briefly, the right lung was thoroughly minced and incubated for $30 \mathrm{~min}$ in digestion medium in a humidified incubator at $37^{\circ} \mathrm{C}$ and $5 \% \mathrm{CO}_{2}$. Organ fragments were resuspended, fresh digestion medium was added, and incubation was extended for $15 \mathrm{~min}$. After a final resuspension, samples were centrifuged and resuspended in calcium and magnesium-free PBS containing $10 \mathrm{mM}$ EDTA at room temperature. Finally, the cells were subjected to red blood cell lysis, washed in fluorescence-activated cell sorter-EDTA, passed through a $50 \mu \mathrm{m}$ cell strainer, and kept on ice until labelling. Cell counting was performed with a Z2 Beckman-Coulter particle counter (Beckman-Coulter, Ghent, Belgium).

\section{Labelling of BAL cells and lung single cell suspensions for flow cytometry}

Flow cytometric analysis of DCs, macrophages, granulocytes, T- and B-lymphocytes was defined as detailed previously [16]. Monoclonal antibodies used to identify mouse DC populations were biotinylated anti-CD11c and phycoerythrin (PE)-conjugated anti-IA ${ }^{\mathrm{b}}$. Additional markers used for DC phenotyping were PE-conjugated CD40, CD86/B7-2, CD80/ B7-1 and CD54/intercellular cell adhesion molecule (ICAM)-1. Isotype controls were rat immunoglobulin (Ig)- $\mathrm{G}_{2 a}$, rat $\operatorname{IgG}_{2 b}$ and Armenian hamster IgG. Markers used for mouse T-cell subpopulations staining were CD4, CD8, CD3 and CD69 (activation marker). Granulocytes were stained with GR1 and B-cells were revealed as CD19 positive. All monoclonal antibodies were obtained from BD Pharmingen (Becton Dickinson Pharmingen, Erembodegem, Belgium), except antiCD54 (Research Diagnostic Inc., Flanders, NJ, USA) and antiCD11c (N418 hybridoma; gift from M. Moser, Brussels Free University, Belgium).

Flow cytometry data acquisition was performed on a duallaser FACS Vantage ${ }^{\mathrm{TM}}$ flow cytometer running CELLQuest ${ }^{\mathrm{TM}}$ software (Becton Dickinson, Mountain View, CA, USA). FlowJo software (www.Treestar.com) was used for data analysis.

\section{Histology and morphometric analysis}

After excision of the right lung, the left lung was fixated by gentle infusion of fixative ( $4 \%$ paraformaldehyde) through the tracheal cannula by a continuous release pump under pressure and volume-controlled conditions $\left(12 \mathrm{~mL} \cdot \mathrm{h}^{-1} ; 10 \mathrm{~min}\right)$. After excision, the lung was immersed in fresh fixative for a period of $4 \mathrm{~h}$. The lung lobe was embedded in paraffin and cut in $3 \mu \mathrm{m}$ transversal sections. Tissue samples were stained with haematoxylin and eosin (Klinipath, Geel, Belgium). For each animal, 10 fields at a magnification of $\times 200$ were captured in a blinded fashion using a Zeiss KS400 image analyser platform (Zeiss, Oberkochen, Germany).

Emphysema is a structural disorder characterised by damage to the lung parenchyma. The destruction of the alveolar walls will lead to enlargement of the alveolar spaces. The destruction and the enlargement processes may proceed chronologically or simultaneously $[17,18]$. Therefore, it is recommended to evaluate both parameters to quantify emphysematous lesions [19].

Quantification of airspace enlargement was determined after 6 months smoke exposure by mean linear intercept $(\mathrm{Lm})[20,21]$ and mean alveolar surface $(\mathrm{Am})$. The measurement of $\mathrm{Lm}$ was performed by means of a $100 \times 100 \mu \mathrm{m}$ grid passing randomly through the lung. The total length of each line of the grid divided by the number of alveolar intercepts gave the average distance between alveolated surfaces, or the Lm. The same image was used for the measurement of the Am. An alveolus or airspace is defined as the space surrounded by the alveolar wall, which in case of an alveolus opening onto a duct ends at the mouth of the alveolus. The cross-sectional surface of the airspace was calculated and divided by the number of alveoli to obtain Am.

The destruction of alveolar walls was quantified by the destructive index (DI) [17]. Briefly, a grid with 42 points 
which were at the centre of hairline crosses was superimposed on the lung field. Structures lying under these points were classified as normal (N) or destroyed (D) alveolar and/or duct spaces. Points falling over other structures, such as duct walls, alveolar walls, etc. did not enter into the calculations. The DI was calculated from the formula:

$$
\mathrm{DI}=\mathrm{D} /(\mathrm{D}+\mathrm{N}) \times 100
$$

Morphometric quantification of DC infiltration in lung tissue was performed using a double immunofluorescent staining protocol, as detailed previously [22]. DCs, identified as small elongated dendritic-like major histocompatability complex (MHC) $\mathrm{II}^{+} \mathrm{B} 220^{-}$cells, were counted in the tissue area surrounding the airway epithelium and accompanying blood vessel(s), as well as the lung parenchyma. To quantify DC infiltration, 10 airways per mouse were examined. Results are expressed as cell counts relative to tissue surface $\left(\mathrm{mm}^{2}\right)$.

\section{Statistical analysis}

Reported values were expressed as mean $\pm \mathrm{SE}$ of the mean \pm SEM. Cellular composition of BAL, DCs and T-lymphocytes in the lungs, alveolar airspace size (Lm, Am) and alveolar wall destruction (i.e. DI) for different groups were
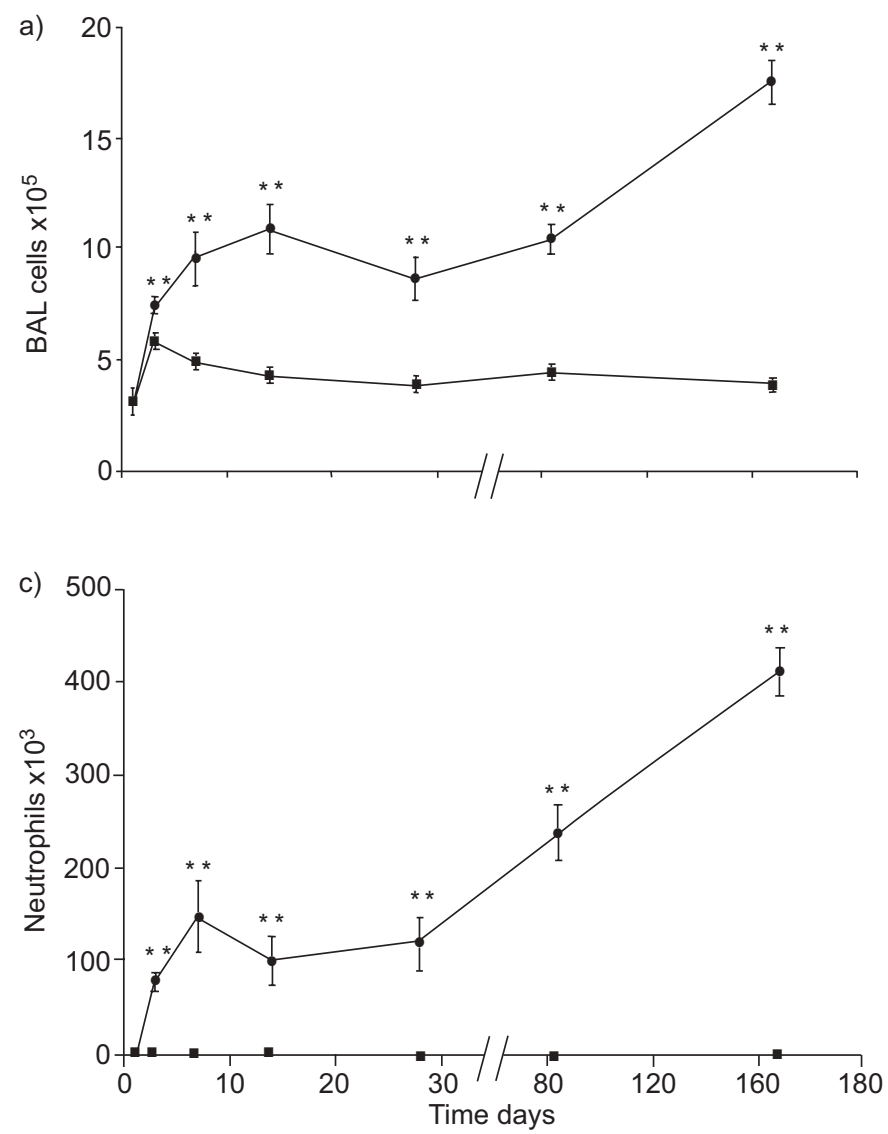

compared via the Kruskal-Wallis test for multiple comparisons. When significant differences were observed, pair wise comparisons were made using the Mann-Whitney U-test with Bonferroni corrections. A p-value $<0.05$ was considered significant.

\section{RESULTS \\ Inflammatory changes in BAL fluid}

In order to investigate the effects of smoke exposure on airway and lung inflammation, the three main pulmonary compartments of air or smoke-exposed mice were studied; the airway and alveolar lumen (by BAL), the airway wall, and the lung parenchyma (by histology and immunohistochemistry on lung tissue sections, and by flow cytometry on cell suspensions of lung digests). The experiments were performed in triplicate.

Tobacco-exposed mice developed a progressive biphasic increase in the total number of inflammatory cells in BAL. After 3 days of smoke exposure, a significant increase of total BAL cells was observed $(p=0.008)$. By the end of 6 months, a 4.5-fold increase of total BAL cells in CSexposed mice compared with air-exposed mice was observed $(\mathrm{p}<0.001$; fig. 1a). This increase was due to an accumulation of monocytes/macrophages, neutrophils, lymphocytes and DCs in BAL fluid (figs 1 and 2). The highest relative
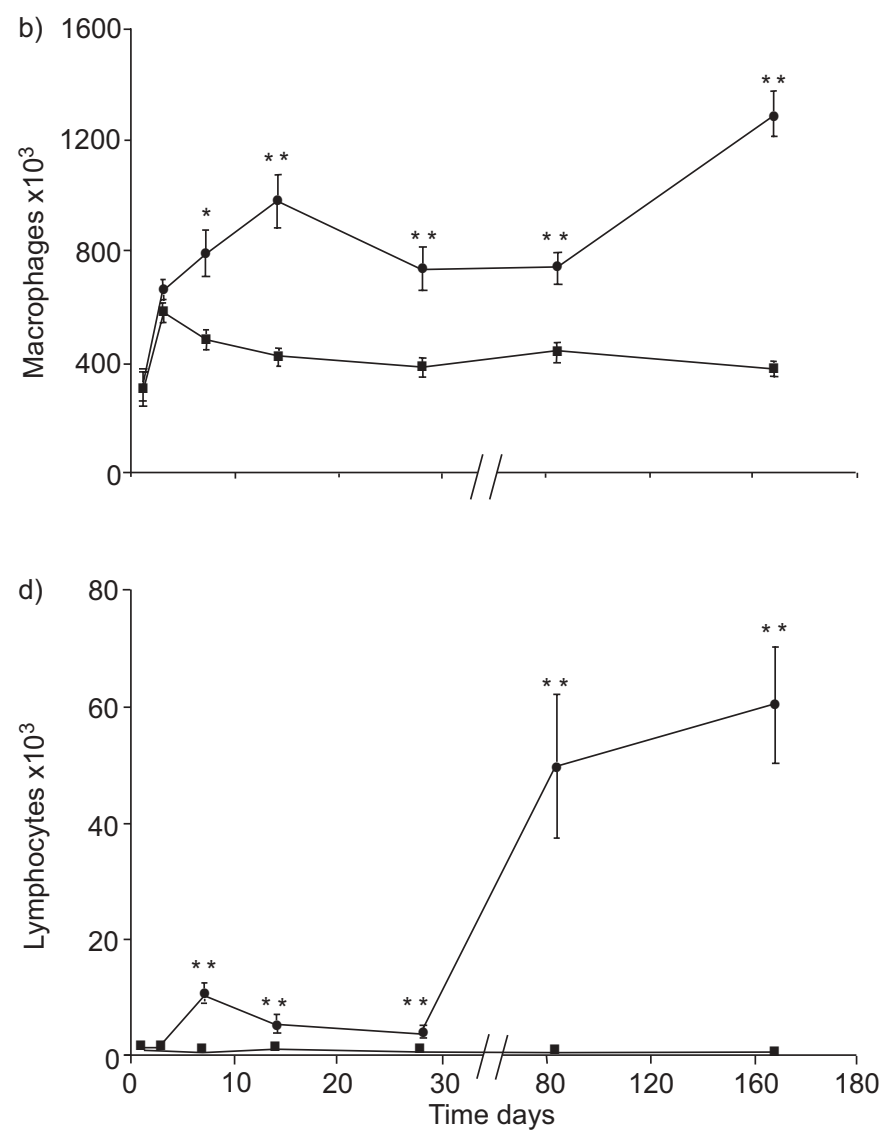

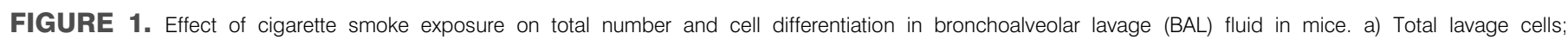
b) macrophages; c) neutrophils; and d) lymphocytes. : smoke-exposed mice; : air-exposed mice. Animals (at days 1, 3, 7 and 14 days, n=6; at weeks 4, 12 and 24,

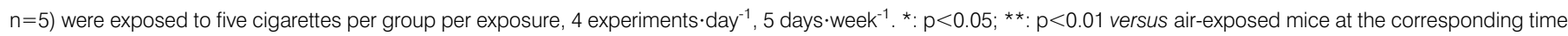
point). Experiments were performed in triplicate. Combined data from the three independent experiments are shown. 

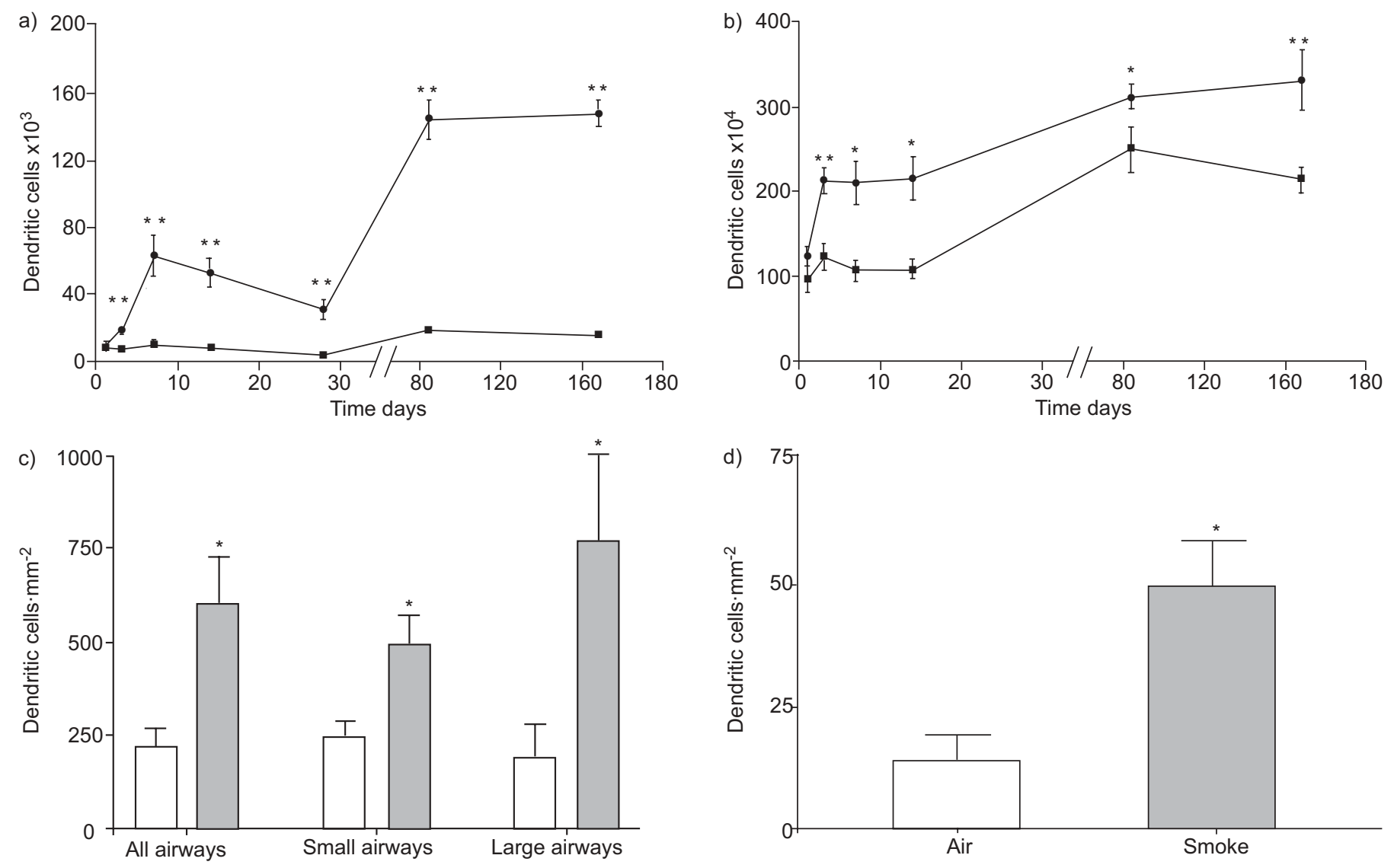

FIGURE 2. Time course of the effect of cigarette smoke exposure on the absolute number of dendritic cells in a) bronchoalveolar lavage fluid, and b) lung parenchyma of smoke-exposed mice $(\bullet)$ and air-exposed mice (-). Effect of 24 weeks cigarette smoke exposure on the number of dendritic cells $\mathrm{c}$ ) surrounding the airway wall, or d) present in the lung parenchyma in air-exposed mice $(\square)$ and smoke-exposed mice ( $\square$ ). Animals (at days 1, 3, 7 and 14 days $n=6$; at weeks 4,12 and $24 n=5$ ) were exposed to five cigarettes per group per exposure, 4 experiments day $^{-1}, 5$ days $\cdot$ week $^{-1}$. $*: p<0.05 ; * *: p<0.01$ versus air-exposed mice at corresponding time point. Experiments were performed in triplicate. Combined data from the three independent experiments are shown.

increase over time was seen for neutrophils, DCs and lymphocytes.

Morphological analysis on cytospins revealed a slight, but significant, increase of the total number of monocytes/ macrophages in BAL after 7 days of smoke exposure $(\mathrm{p}=$ 0.01). A marked increase was observed at 6 months (total number of macrophages at 6 months: smoke $=$ $1289.3 \pm 80.1 \times 10^{3} \quad$ versus air $=381.4 \pm 27.7 \times 10^{3} ; \quad \mathrm{p}<0.001$; fig 1b). Flow cytometry confirmed the observed increase of BAL macrophage numbers after smoke exposure (data not shown).

On cytospins, neutrophils were almost absent from the BAL of air-exposed animals. Smoke-exposed animals developed a progressive BAL neutrophilia. The number of neutrophils was significantly increased after 3 days $(p<0.001)$ and increased further up to 6 months (total number of neutrophils at 6 months: smoke $=412.2 \pm 24.8 \times 10^{3}$ versus air $=0.2 \pm 0.1 \times 10^{3} ; \mathrm{p}<0.001$; fig 1c). Eosinophil numbers in BAL were small in both groups and did not change significantly over time (data not shown).

Smoke-exposed animals developed a progressive increase of T-lymphocytes in the airways (as enumerated on cyto centrifuge preparations). The amount of lymphocytes was significantly increased at 7 days $(p=0.004)$ and increased further up to 6 months (total number of lymphocytes at 6 months: smoke $=60.3 \pm 10.0 \times 10^{3}$ versus air $=0.7 \pm 0.2 \times 10^{3}$; $\mathrm{p}<0.001$; fig $1 \mathrm{~d}$ ).

A few B-lymphocytes in BAL were present in baseline conditions. After 3 days smoke exposure, B-lymphocytes were significantly increased compared with air-exposed mice $(\mathrm{p}=0.004$; data not shown).

\section{Increased number of dendritic cells in BAL and lungs}

The elevated number of monocytes/macrophages in BAL following smoke exposure made the current authors curious as to whether the DCs were originally derived from monocyte 
precursors in the bone marrow [23], and if playing an important role in the pulmonary immune responses would increase.

DCs were identified by flow cytometry using two simultaneous immunofluorescent criteria, which were low autofluorescence and intermediate-to-high levels of CD11c [24]. Smokeexposed mice showed a significant and biphasic increase in DCs in lavage fluid. After 3 days smoke exposure, DCs in BAL were already increased $(\mathrm{p}<0.001)$. By the end of 6 months, a 10 -fold increase of these cells was observed (total number of BAL DCs at 6 months: smoke $=148.5 \pm 8.0 \times 10^{3}$ versus air $=15.8 \pm 0.8 \times 10^{3} ; \mathrm{p}<0.001$; fig $\left.2 \mathrm{a}\right)$.

Also in the lung parenchyma, tobacco smoke induced a slight, but significant increase in DC numbers after 3 days of exposure $(\mathrm{p}=0.005)$. At 6 months, lung parenchymal DC numbers increased 1.5-fold (total number of lung DCs at 6 months: smoke $=332.6 \pm 35.9 \times 10^{4}$ versus air $=214.6 \pm 15.4 \times 10^{4} ; \mathrm{p}=0.009$; fig 2b).

DCs were also identified by immunohistochemistry based upon a combination of morphology, MHC II positivity and the absence of the B-cell marker B220 using a double immunofluorescent staining protocol. CS induced a significant increase of DCs in the tissue area surrounding both small $(0-1000 \mu \mathrm{m}$ airway perimeter) and large airways (1000-2000 $\mu \mathrm{m}$; fig 2c). Thus, the DC infiltration in the airway wall was not dependent upon airway size $(p=0.65)$. There was also a significant accumulation of DCs in lung parenchyma in tobacco exposed animals compared with air-exposed mice (fig $2 \mathrm{~d}$ and fig 3).

The activation status of pulmonary DCs in smoke-exposed mice was assessed by examining cell surface expression of MHC II and T-cell costimulatory molecules. Both in BAL and in lung parenchyma, high expression of CD11 (CD11chi) low autofluorescent DCs of tobacco exposed mice had elevated levels of MHC II and the costimulatory molecule CD40, compared with DCs of air-exposed animals. Moreover, smoke exposure also increased the expression of the costimulatory molecule CD86 (B7.2) on DCs when present in the BAL compartment (fig 4).

\section{Inflammatory changes in the lungs}

The total number of lung cells did not change significantly over time (data not shown). Flow cytometric analysis of lung digests demonstrated increased numbers of macrophages and CD4+ and CD8+ T-lymphocytes in the smoke-exposed animals (fig 5).

Pulmonary macrophages were identified by flow cytometry as $\mathrm{CD}_{11 \mathrm{c}^{+}}$high autofluorescent cells. In contrast to low autofluorescent DCs, these cells have typical macrophage morphology and were MHC II low to negative [24]. In the lungs of control mice, the high autofluorescent $\mathrm{CD}_{11 \mathrm{c}^{+}}$cells declined with age, whereas in smoke-exposed mice macrophage numbers increased significantly after 12 weeks $(p=0.009)$ compared with control mice. This difference persisted over time (total number of macrophages at 6 months: smoke $=226.8 \pm 27.4 \times 10^{4}$ versus air $=75.4 \pm 3.9 \times 10^{4} ; \mathrm{p}=0.009$; fig 5a).

The progressive accumulation of DCs with high expression of T-cell costimulatory molecules prompted examination of the extent, phenotype and activation status of T-cell populations in smoke-exposed lungs. The lung T-lymphocyte subsets population did not change significantly after shorttime smoke exposure. In contrast, after 12 weeks the total number of lymphocytes was significantly increased $(p=0.009)$ and increased further up to 6 months (total number of CD3+ lymphocytes in the lungs at 6 months: smoke= $414.5 \pm 48.3 \times 10^{4} \quad$ versus air $\left.=261.4 \pm 11.5 \times 10^{4} ; \quad \mathrm{p}=0.014\right)$ Smoke exposure induced a significant increase in both CD4+ and CD8+ T-cells compared with air-exposed mice. Total number of CD4+ T-lymphocytes in the lungs at 6 months:
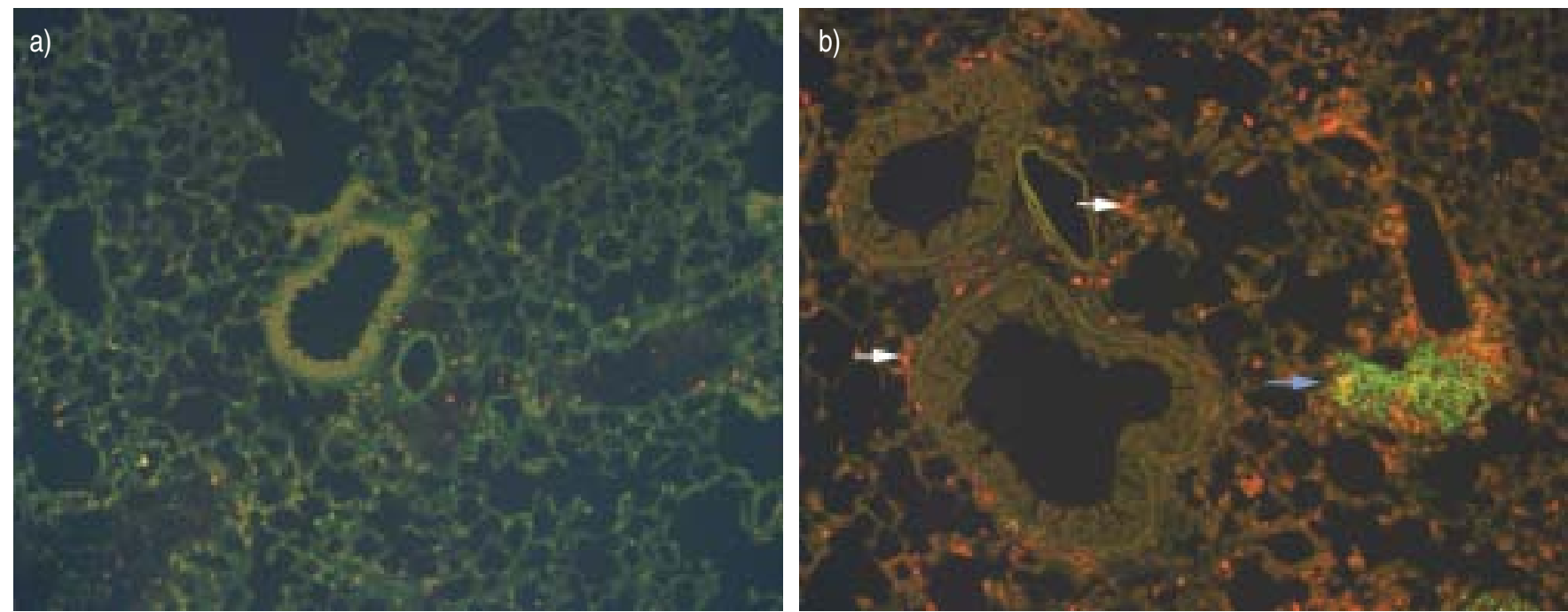

FIGURE 3. Photomicrographs of a double immunofluorescent staining of lung tissue of a) air-exposed, and b) smoke-exposed mice at 24 weeks. Dendritic cells (white arrows) were identified based upon a combination of morphology, major histocompatability complex-II positivity (red stain) and the absence of the B-cell marker B220 (green stain). Blue arrow indicates a B-cell follicle. 

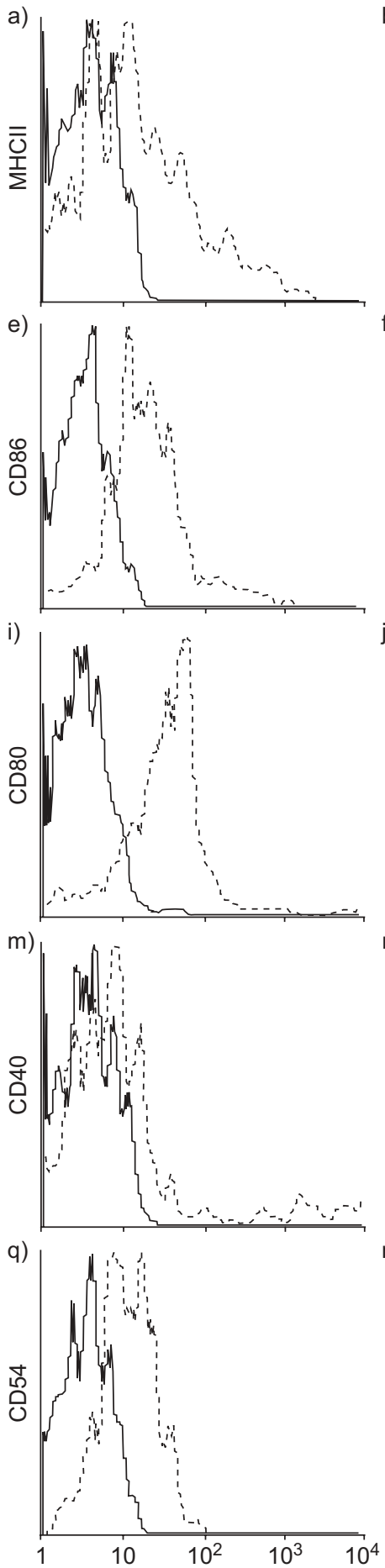
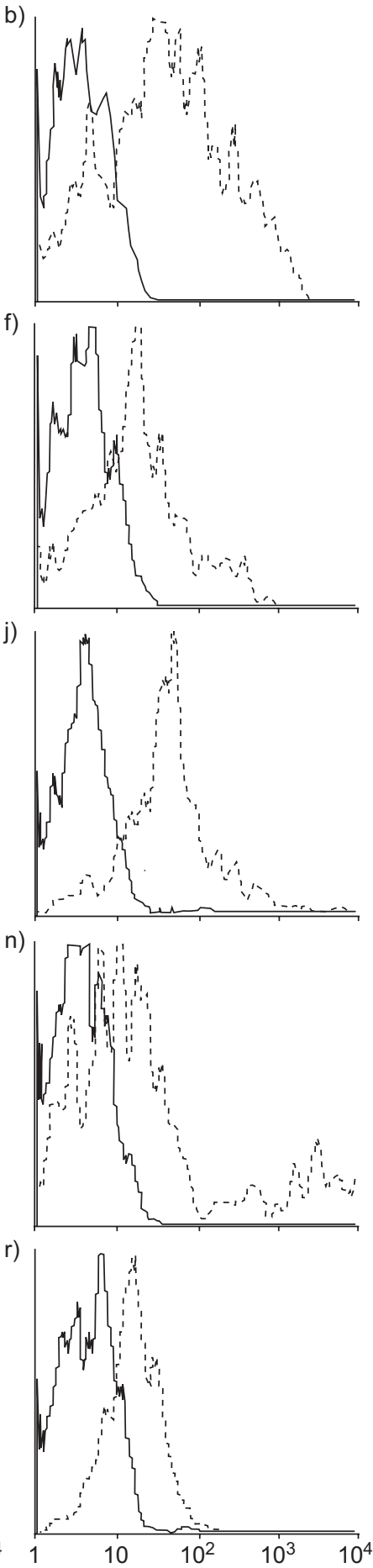
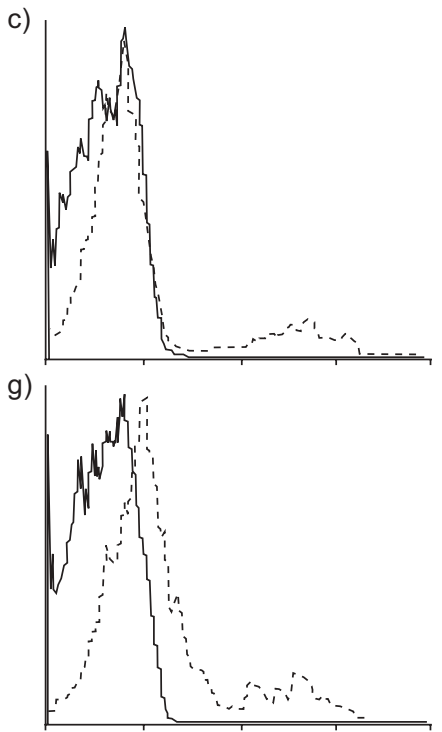

k)

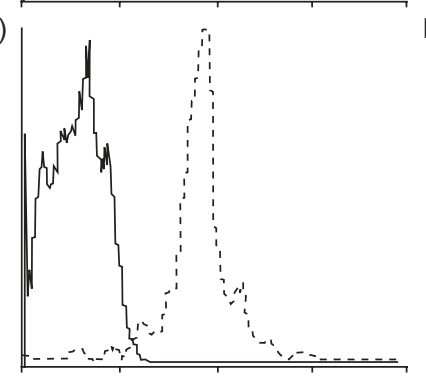

o)

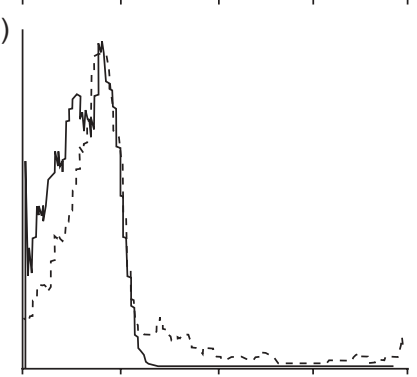

s)

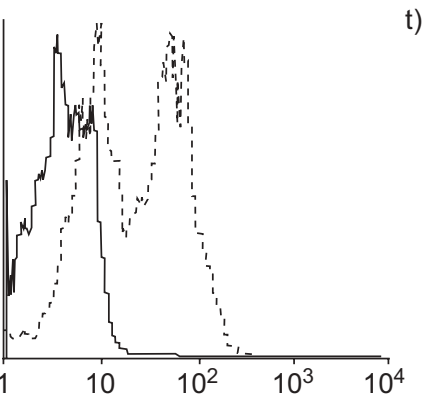

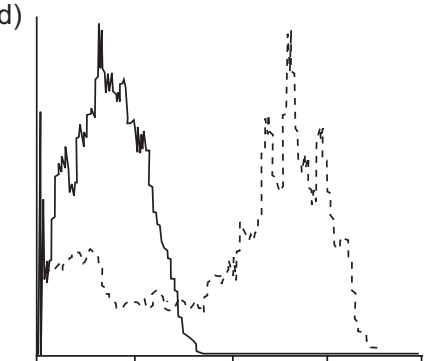

h)
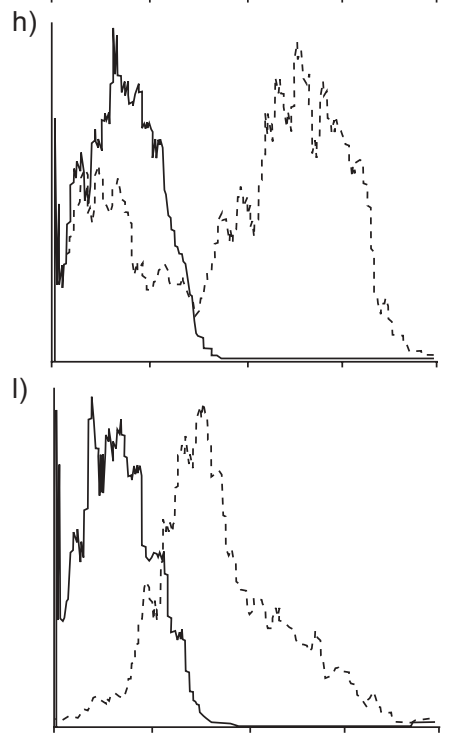

p)

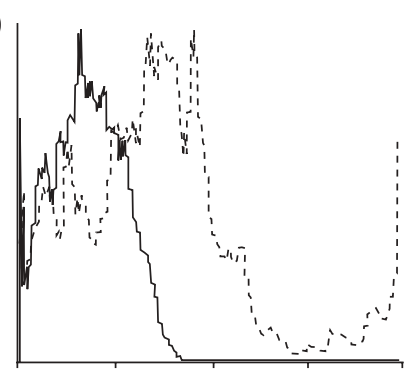

)

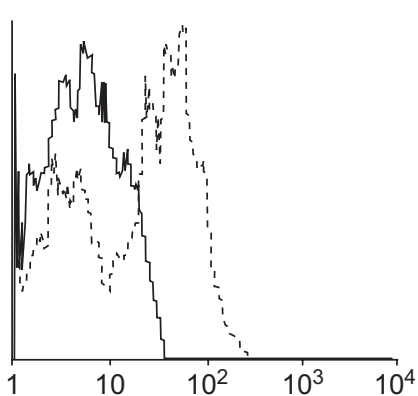

FIGURE 4. Phenotypic flow cytometric analysis of dendritic cells identified in the lungs (a, b, e, f, l, j, m, n, $q, r$ ) and bronchoalveolar lavage (BAL) (c, $d, g$, $h, k, l, o, p, s, t)$ in air and smoke-exposed mice for 4 weeks. Compared with air-exposed dendritic cells (a, c, e, g. i, k, m, o, q and s), dendritic cells of smoke-exposed mice $(b, d, f, h, j, l, n, p, r$ and t) had upregulated levels of major histocompatability complex (MHC) II, CD86 and CD40 in BAL; and upregulated levels of MHC II and CD40 in lung tissue. - - -: specific marker staining; _ - isotype control staining. The data shown are from one animal and from one experiment, representative of two independent experiments.

smoke $=229.3 \pm 36.2 \times 10^{4}$ versus air $=103.7 \pm 6.7 \times 10^{4}(\mathrm{p}=0.014)$. Total number of CD $8+$ T-lymphocytes in the lungs at 6 months: smoke $=157.0 \pm 35.3 \times 10^{4}$ versus air $=110.3 \pm 4.2 \times 10^{4} \quad(p=0.05$; fig $5 \mathrm{~b}$ and $5 \mathrm{c})$.
In addition, activation of $\mathrm{CD} 3+\mathrm{CD} 4+$ and $\mathrm{CD} 3+\mathrm{CD} 8+\mathrm{T}$-cells was examined. After 14 days smoke exposure, an increased number of activated CD4+ and CD8+ T-cells was present in lung digests. At 24 weeks the early activation marker CD69 

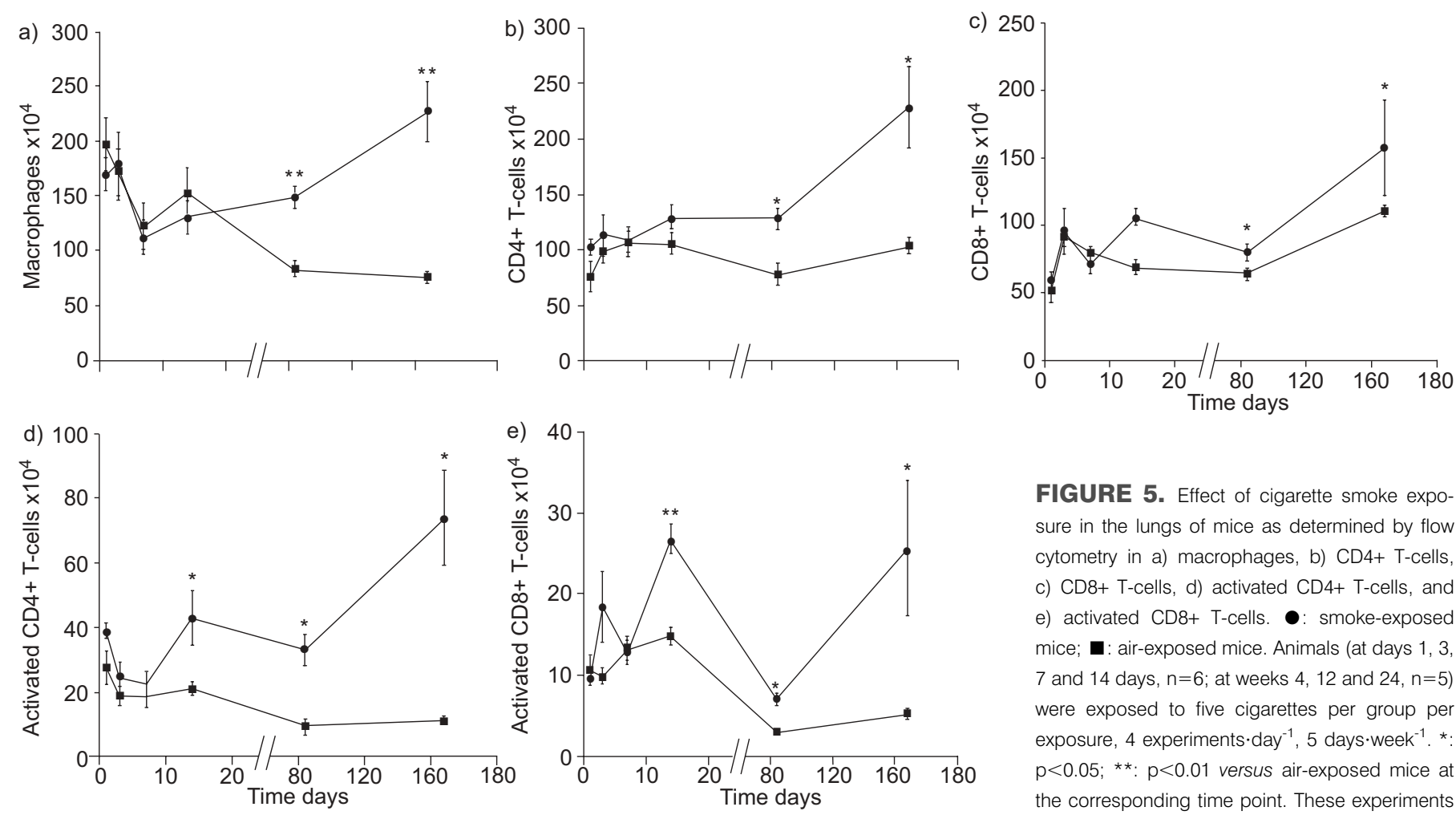

\begin{abstract}
FIGURE 5. Effect of cigarette smoke exposure in the lungs of mice as determined by flow cytometry in a) macrophages, b) CD4+ T-cells, c) CD8+ T-cells, d) activated CD4+ T-cells, and e) activated CD8+ T-cells. - : smoke-exposed mice; : air-exposed mice. Animals (at days 1, 3, 7 and 14 days, $n=6$; at weeks 4,12 and $24, n=5$ ) were exposed to five cigarettes per group per exposure, 4 experiments $\cdot$ day $^{-1}, 5$ days $\cdot$ week $^{-1}$. * $p<0.05 ; * *: p<0.01$ versus air-exposed mice at the corresponding time point. These experiments were performed in triplicate. The results of one representative experiment are shown.
\end{abstract}

was expressed in $31.82 \pm 1.37 \%$ CD4+ T-cells and $15.53 \pm 1.79 \%$ CD8+ T-cells in the smoke-exposed group, compared with $11.09 \pm 0.36 \% \quad(p=0.014)$ and $4.65 \pm 0.60 \% \quad(p=0.014)$, respectively in the air-exposed group (figs $5 \mathrm{~d}$ and $5 \mathrm{e}$ ).

Flow cytometric analysis revealed no differences in granulocytes in the lungs between smoke-exposed animals and airexposed mice (data not shown).

In the lungs, no differences were observed in the B-cell population (data not shown).

\section{Histology}

Emphysema is a structural disorder characterised by destruction of the alveolar walls and enlargement of the alveolar spaces. Destruction of alveolar walls was determined by measuring the DI and enlargement of alveolar spaces by quantifying the $\mathrm{Lm}$ and the Am. Microscopic analysis of lung tissue sections revealed a significant degree of emphysema after 6 months of smoke exposure (fig. 6). Tobacco smoke clearly induced alveolar wall destruction. The DI for the air-exposed mice ranged from 0.0-9.8 (mean $\pm \mathrm{SEM}=4.2 \pm 0.5$ ), while the DI for the smoke-exposed animals ranged from 20.2-35.1 (mean \pm SEM $=25.8 \pm 0.7 ; \mathrm{p}<0.001$ versus control group). Furthermore, exposure to CS significantly induced airspace enlargement. The $\mathrm{Lm}$ was $23 \%$ higher in CS-exposed mice compared with air-exposed mice $(\mathrm{Lm}$ at 6 months smoke $=42.2 \pm 0.20 \mu \mathrm{m}$ versus air $=34.3 \pm$ $0.21 \mu \mathrm{m} ; \mathrm{p}<0.001)$. A significant increase of Am was also observed in smoke-exposed animals (Am at 6 months: smoke $=$ $783.4 \pm 79.8 \mu \mathrm{m}^{2}$ versus air $\left.=511.8 \pm 42.41 \mu \mathrm{m}^{2} ; \mathrm{p}=0.015\right)$.

\section{DISCUSSION}

In the present study, the time course of the changes in the major inflammatory cells was investigated both in the airways and the lungs of mice during 6 months of exposure to CS, up until the development of pulmonary emphysema. In response to CS, a progressive biphasic infiltration of inflammatory cells, including DCs, neutrophils, macrophages, T- and B-lymphocytes, was observed in the BAL compartment of mice. DCs, macrophages, and lymphocytes, but not neutrophils, also accumulated in the lungs following smoke exposure. Moreover, after 6 months CS exposure, a significant degree of alveolar destruction developed.

The time course of the different cell types in BAL fluid showed a relatively earlier influx of DCs, neutrophils and macrophages compared with lymphocytes. This suggests that chronic exposure of the airways to CS results in a mixture of acute and chronic inflammatory changes $[3,6]$. The current results can be explained both by an innate and an adaptive immune response to CS, since all cell types that were augmented occur in both types of immune response.

A progressive smoke induced DC recruitment and DC activation was observed in three main pulmonary compartments. These were the bronchoalveolar lumen, the airway wall and the lung parenchyma. To pinpoint DCs in BAL and lung parenchyma, the current authors used a strategy based on the work by HAVENITH et al. [25], who used the bright autofluorescence of alveolar macrophages to separate them efficiently from monocytes and DCs. This DC isolation procedure was further elaborated and demonstrated that 

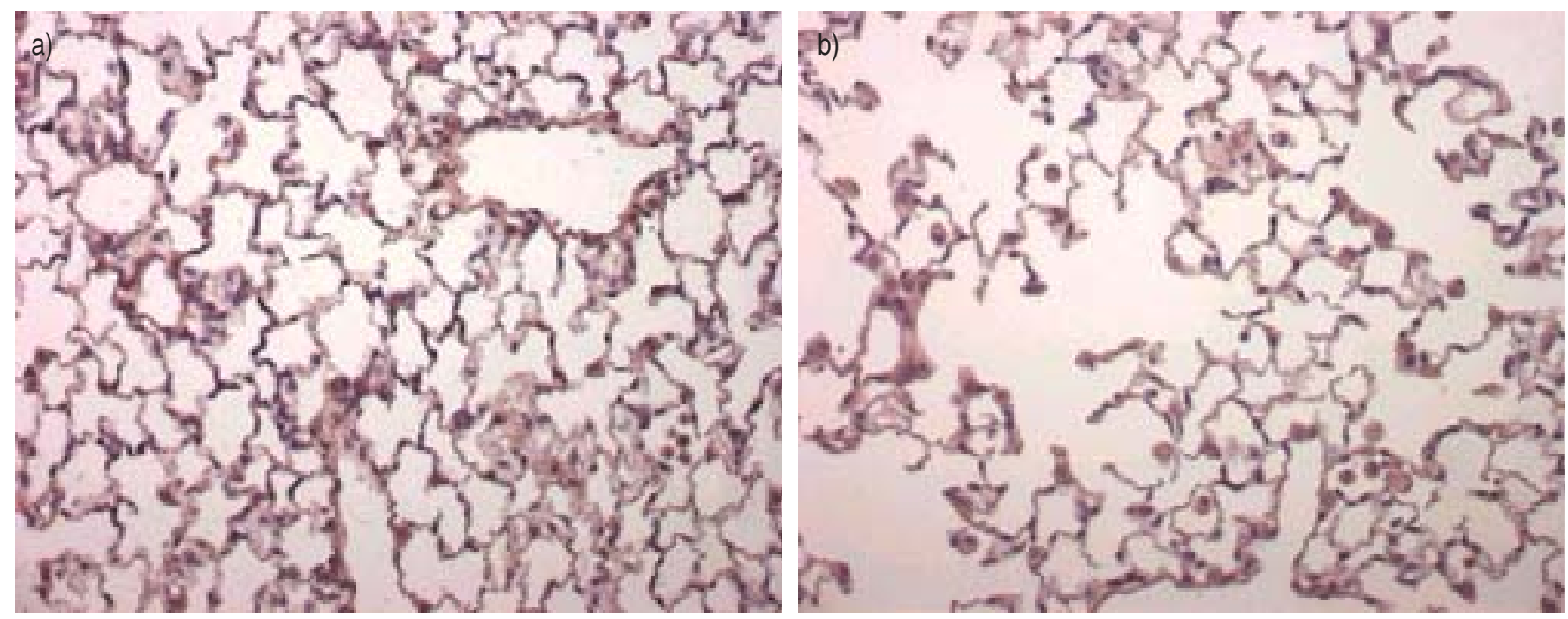

FIGURE 6. Photomicrographs of haematoxylin and eosin stained lung tissue of a) air-exposed and b) smoke-exposed mice at 24 weeks. Smoke exposure clearly induced alveolar wall destruction and airspace enlargement in mice.

DCs and monocytic DC precursors in BAL and lung parenchyma were within the CD11c+ gate of the low autofluorescent cell fraction [24]. The selected cells expressed high levels of CD11c, CD11b and MHC II and, thus, may be considered as myeloid DCs and/or their immediate precursors. Plasmacytoid DCs expressing low levels of CD11c and no $\mathrm{CD} 11 \mathrm{~b}$, were found to be very rare in the lungs of mice exposed either to air or to CS (data not shown). The increase in DCs, both in the BAL compartment and lung parenchyma, was confirmed by immunohistology. The smoke induced DCs were in an activated state as illustrated by an upregulation of MHC II and T-cell costimulatory molecules, CD40 and CD86. The current findings are in agreement with CASOLARO et al. [26], who reported an accumulation of Langerhans cells (LC), a cell type now known to be a subset of DCs, in BAL fluid of smokers. SOLER et al. [27] also observed an increase of LC in smokers' lungs. Others studies have suggested a relationship between CS inhalation and lung interstitial granulomas containing large numbers of LC in pulmonary LC histiocytosis [28]. This LC granulomatosis was correlated with parenchymal lung destruction. In a short-term experiment the density of pulmonary LCs was increased in tobacco smoke-exposed mice and was associated with histopathological changes in the lung characteristic of LC granulomatosis [15]. In contrast to the present study and previous other studies, a recent report by RoBBINS et al. [29] described a decrease in pulmonary DC number and activation following tobacco smoke exposure, impairing the immune response against adenoviral infection. The reason for these divergent results is not clear, but this could be due to differences in the flow cytometric methodology or the intensity of smoke exposure (low dose =two cigarettes daily in the study by RoBBINS et al.; high dose $=20$ cigarettes daily in present experiments). Future studies are needed to clarify whether the effects of CS on DCs are dose-dependent.

The elevated number of pulmonary DCs following smoke exposure suggests a possible role for this cell in the pathogenesis of COPD. It is now clear that the DC plays a major role in the innate immune response and is the most powerful antigenpresenting cell in the respiratory tract. The DC network within and under the epithelium of the conducting airways is ideally positioned to perform a sentinel role against toxic inhalants. The current authors hypothesised that tobacco smoke, containing numerous toxic low molecular weight compounds, activates bronchoalveolar DCs directly. Activated DCs can secrete a whole range of inflammatory chemokines, inducing the recruitment and activation of more DCs and other inflammatory cells [30], such as macrophages, neutrophils, natural killer cells and lymphocytes. Another mechanism of smoke induced DC activation could involve stimulation by heat shock proteins, which are released during cell necrosis [31]. This is supported by in vitro experiments showing that tobacco smoke extract causes dose-dependent apoptosis and necrosis of airway epithelial cells [32].

Proteolytic enzymes are among the numerous mediators released by inflammatory cells after tobacco smoke exposure. Neutrophils and especially macrophages, are a rich source of proteases [33], releasing neutrophil elastase, cathepsins and matrix metalloproteinases upon activation, which can degrade extracellular matrix (ECM) components. The resulting ECM breakdown products (e.g. elastin fragments) have the potential to attract and activate more inflammatory cells, inducing a vicious circle. In this mouse model of chronic CS exposure, neutrophil numbers were increased in the BAL compartment, but not in the lungs. This is in agreement with the observations in patients with COPD, where increased numbers of activated neutrophils were found in sputum and BAL fluid, yet only relatively small increases were observed in the lung parenchyma [34]. The marked increase in the numbers of macrophages in both the BAL and pulmonary compartment of CS-exposed mice is reminiscent of the vast increase of macrophage numbers in sputum, BAL fluid and lung parenchyma in patients with COPD. Macrophages may play a pivotal role in COPD, since they are activated by CS extract and secrete not only elastolytic enzymes, but also many inflammatory chemokines (e.g. interleukin-8 and CXCR3-ligands), attracting 
neutrophils and CD8+ T-lymphocytes, respectively [33]. An age-related decrease in the number of macrophages in the lungs of air-exposed mice was observed. A similar observation was made by SHIBATA et al. [35], where in lungs of control mice Mac-3+ cell numbers declined with increasing age.

Contrary to the human inflammatory response to tobacco smoke, a predominant increase of CD4+ T-cells rather than CD8+ T-cells was observed $[6,36]$. A similar observation was made by both MESHI et al. [37] and VITALIs et al. [38] in guinea pigs where the CD4+ T-cell increase was associated with smoke exposure, whereas the increase in CD8+ T-cells was linked with the presence of viral infection.

Remarkably, the increase of total cells, DCs and macrophages in BAL showed a progressive, but biphasic response. A similar pattern was shown by STEVENSON et al. [39] in a smoke-exposed rat model. Starting from the fourth week of smoke exposures, a 'transcriptional shift' was observed between smoke-exposed and control animals. Associated with this 'shift' were clusters of up- and downregulated genes, including proteases and proinflammatory targets (e.g. matrix metalloprotienase-12), which may be involved in the COPD pathogenesis.

In conclusion, it seems likely that all the different inflammatory cells together are responsible for lung injury caused by cigarette smoke. However, whereas the role of neutrophils and macrophages in the pathogenesis of chronic obstructive pulmonary disease has been clearly highlighted, the marked increase of dendritic cells in the smoke induced lung inflammation prompts further investigation into the potential role of these cells in the pathophysiology of chronic obstructive pulmonary disease. Of course, since this study is primarily descriptive, this hypothesis has to be explored in further studies, including functional studies in animal models of chronic obstructive pulmonary disease.

\section{ACKNOWLEDGMENTS}

The authors would like to thank E. Castrique, C. Snauwaert, K. De Saedeleer, A. Neessen, I. De Borle, M. Mouton, G. Barbier and P. Degryze for their technical assistance.

\section{REFERENCES}

1 Pauwels RA, Buist AS, Calverley PM, Jenkins CR, Hurd SS. Global strategy for the diagnosis, management, and prevention of chronic obstructive pulmonary disease. NHLBI/WHO Global Initiative for Chronic Obstructive Lung Disease (GOLD) Workshop summary. Am J Respir Crit Care Med 2001; 163: 1256-1276.

2 Sutherland ER, Martin RJ. Airway inflammation in chronic obstructive pulmonary disease: comparisons with asthma. J Allergy Clin Immunol 2003; 112: 819-827.

3 Retamales I, Elliott WM, Meshi B, et al. Amplification of inflammation in emphysema and its association with latent adenoviral infection. Am J Respir Crit Care Med 2001; 164 : 469-473.

4 Saetta M, Finkelstein R, Cosio MG. Morphological and cellular basis for airflow limitation in smokers. Eur Respir J 1994; 7: 1505-1515.

5 Jeffery PK. Structural and inflammatory changes in COPD: a comparison with asthma. Thorax 1998; 53: 129-136.
6 Hogg JC, Chu F, Utokaparch S, et al. The nature of smallairway obstruction in chronic obstructive pulmonary disease. N Engl J Med 2004; 350: 2645-2653.

7 Zhang P, Summer WR, Bagby GJ, Nelson S. Innate immunity and pulmonary host defense. Immunol Rev 2000; 173: 39-51.

8 Holt PG. Regulation of antigen-presenting cell function(s) in lung and airway tissues. Eur Respir J 1993; 6: 120-129.

9 Holt PG, Stumbles PA. Regulation of immunologic homeostasis in peripheral tissues by dendritic cells: the respiratory tract as a paradigm. J Allergy Clin Immunol 2000; 105: 421-429.

10 Huang Q, Liu D, Majewski P, et al. The plasticity of dendritic cell responses to pathogens and their components. Science 2001; 294: 870-875.

11 Banchereau J, Steinman RM. Dendritic cells and the control of immunity. Nature 1998; 392: 245-252.

12 Lipscomb MF, Masten BJ. Dendritic cells: immune regulators in health and disease. Physiol Rev 2002; 82: 97-130.

13 Gallucci S, Matzinger P. Danger signals: SOS to the immune system. Curr Opin Immunol 2001; 13: 114-119.

14 Hautamaki RD, Kobayashi DK, Senior RM, Shapiro SD. Requirement for macrophage elastase for cigarette smokeinduced emphysema in mice. Science 1997; 277: 2002-2004.

15 Zeid NA, Muller HK. Tobacco smoke induced lung granulomas and tumors: association with pulmonary Langerhans cells. Pathology 1995; 27: 247-254.

16 Vermaelen KY, Carro-Muino I, Lambrecht BN, Pauwels RA. Specific migratory dendritic cells rapidly transport antigen from the airways to the thoracic lymph nodes. J Exp Med 2001; 193: 51-60.

17 Saetta M, Shiner RJ, Angus GE, et al. Destructive index: a measurement of lung parenchymal destruction in smokers. Am Rev Respir Dis 1985; 131: 764-769.

18 Saito K, Cagle P, Berend N, Thurlbeck WM. The "destructive index" in nonemphysematous and emphysematous lungs. Morphologic observations and correlation with function. Am Rev Respir Dis 1989; 139: 308-312.

19 Robbesom AA, Versteeg EM, Veerkamp JH, et al. Morphological quantification of emphysema in small human lung specimens: comparison of methods and relation with clinical data. Mod Pathol 2003; 16: 1-7.

20 Thurlbeck WM. Measurement of pulmonary emphysema. Am Rev Respir Dis 1967; 95: 752-764.

21 Bartalesi B, Cavarra E, Fineschi S, et al. Different lung responses to cigarette smoke in two strains of mice sensitive to oxidants. Eur Respir J 2005; 25: 15-22.

22 Vermaelen KY, Cataldo D, Tournoy K, et al. Matrix metalloproteinase-9-mediated dendritic cell recruitment into the airways is a critical step in a mouse model of asthma. J Immunol 2003; 171: 1016-1022.

23 Holt PG, Haining S, Nelson DJ, Sedgwick JD. Origin and steady-state turnover of class II MHC-bearing dendritic cells in the epithelium of the conducting airways. J Immunol 1994; 153: 256-261.

24 Vermaelen K, Pauwels R. Accurate and simple discrimination of mouse pulmonary dendritic cells and macrophages population by flow-cytometry: methodology and new insights. Cytometry 2004; 61A: 170-177.

25 Havenith CE, Breedijk AJ, van Miert PP, et al. Separation of alveolar macrophages and dendritic cells via 
autofluorescence: phenotypical and functional characterization. J Leukoc Biol 1993; 53: 504-510.

26 Casolaro MA, Bernaudin JF, Saltini C, Ferrans VJ, Crystal RG. Accumulation of Langerhans' cells on the epithelial surface of the lower respiratory tract in normal subjects in association with cigarette smoking. Am Rev Respir Dis 1988; 137: 406-411.

27 Soler P, Moreau A, Basset F, Hance AJ. Cigarette smokinginduced changes in the number and differentiated state of pulmonary dendritic cells/Langerhans cells. Am Rev Respir Dis 1989; 139: 1112-1117.

28 Tazi A, Moreau J, Bergeron A, Dominique S, Hance AJ, Soler P. Evidence that Langerhans cells in adult pulmonary Langerhans cell histiocytosis are mature dendritic cells: importance of the cytokine microenvironment. J Immunol 1999; 163: 3511-3515.

29 Robbins CS, Dawe DE, Goncharova SI, et al. Cigarette smoke decreases pulmonary dendritic cells and impacts anti-viral immune responsiveness. Am J Respir Cell Mol Biol 2004; 30: 202-211.

30 Kantengwa S, Jornot L, Devenoges C, Nicod LP. Superoxide anions induce the maturation of human dendritic cells. Am J Respir Crit Care Med 2003; 167: 431-437.

31 Srivastava P. Interaction of heat shock proteins with peptides and antigen presenting cells: chaperoning of the innate and adaptive immune responses. Annu Rev Immunol 2002; 20: 395-425.

32 Vayssier M, Banzet N, Francois D, Bellmann K, Polla BS. Tobacco smoke induces both apoptosis and necrosis in mammalian cells: differential effects of HSP70. Am J Physiol 1998; 275: L771-L779.

33 Barnes PJ, Shapiro SD, Pauwels RA. Chronic obstructive pulmonary disease: molecular and cellular mechanisms. Eur Respir J 2003; 22: 672-688.

34 Finkelstein R, Fraser RS, Ghezzo H, Cosio MG. Alveolar inflammation and its relation to emphysema in smokers. Am J Respir Crit Care Med 1995; 152: 1666-1672.

35 Shibata Y, Zsengeller Z, Otake K, Palaniyar N, Trapnell BC. Alveolar macrophage deficiency in osteopetrotic mice deficient in macrophage colony-stimulating factor is spontaneously corrected with age and associated with matrix metalloproteinase expression and emphysema. Blood 2001; 98: 2845-2852.

36 Saetta M, Di Stefano A, Turato G, et al. CD8+ Tlymphocytes in peripheral airways of smokers with chronic obstructive pulmonary disease. Am J Respir Crit Care Med 1998; 157: 822-826.

37 Meshi B, Vitalis TZ, Ionescu D, et al. Emphysematous lung destruction by cigarette smoke - The effects of latent adenoviral infection on the lung inflammatory response. Am J Respir Cell Mol Biol 2002; 26: 52-57.

38 Vitalis TZ, Kern I, Croome A, Behzad H, Hayashi S, Hogg JC. The effect of latent adenovirus 5 infection on cigarette smoke-induced lung inflammation. Eur Respir J 1998; 11: 664-669.

39 Stevenson CS, Coote K, Winny C, et al. Acute and chronic models of cigarette smoke-induced lung injury in rat. Am J Respir Cell Mol Biol 2005; 169: A205. 